\title{
Régularité des Ondes Élastiques dans la Région Glancing des Ondes $P$
}

\author{
par \\ Tatsushi MORIOKA*
}

\section{Résumé}

Dans cet article, nous montrons que les solutions sortantes de l'équation élastique isotrope avec la condition de Dirichlet au bord possèdent la régularité Gevrey 3 dans la région Glancing des ondes longitudinaux, lorsque la dimension de l'espace est 3, le domaine est extérieur et la courbure Gaussienne du bord est strictement positive. C'est une analogie du travail par Lebeau [14] concernant l'équation des ondes, pour l'équation élastique isotrope.

\begin{abstract}
In this paper, we show that the outgoing solutions of the isotropic elastic equation with Dirichlet condition at the boundary have the Gevrey 3 regularity in the glancing region of the longitudinal waves, when the dimension of the space is 3 , the domain is exterior and the Gaussian curvature is positive. It is an analogy of the work by Lebeau [14] concerning the wave equation, for the isotropic elastic equation.
\end{abstract}

\section{§ 0. Introduction et Résultat}

Soit $\Omega \subset \mathbf{R}^{3}$ un domaine extérieur. On suppose les hypothèses suivantes. (H.1) $\partial \Omega$ est analytique.

(H.2) $\quad \mathbf{R}^{3} \backslash \Omega$ est strictement convexe, c.-à-d. la courbure Gaussienne de $\partial \Omega$ est strictement positive.

Soit $A\left(\partial_{1}\right)$ l'opérateur différentiel dont la valeur est $3 \times 3$ matrice:

$$
A\left(\partial_{1}\right)=\mu_{0} \Delta+\left(\lambda_{0}+\mu_{0}\right) \operatorname{grad} \operatorname{div} \text { dans } \mathbf{R}_{1}^{3},
$$

où $\lambda_{0}, \mu_{0}$ sont constantes vérifiant $\mu_{0}>0,3 \lambda_{0}+2 \mu_{0}>0$ et que $\Delta$ est Laplacien en $\mathbf{R}^{3}$. On définit $L$ par $L=\partial_{t}^{2}-A\left(\partial_{1}\right)$. En notant $\sigma\left(\Delta_{\partial \Omega}\right)$ le symbole

Communiqué par T. Kawai, le 27, novembre, 1998. Revu le 27, avril, 1999.

1991 Mathematics Subject Classification(s): 58G17, 35B65

* Department of Mathematics, Osaka University, Osaka 560-0043, Japan

e-mail: morioka@math.sci.osaka-u.ac.jp 
principal de Laplacien en $\partial \Omega$, on définit $q \in C^{\infty}\left(T^{*}(\mathbb{R} \times \partial \Omega), \mathbb{R}\right)$ par

$$
q(\tau, Z)=-\tau^{2}-c_{1}^{2} \sigma(\Delta \partial \Omega)(Z): \quad \tau \in \mathbb{R}, \quad Z \in T^{*}(\partial \Omega),
$$

où $c_{1}=\left(\lambda_{0}+2 \mu_{0}\right)^{1 / 2}$.

On fixe $T>0, \varepsilon>0$ qui sont suffisamment petites, $z \in \partial \Omega$ et $W_{0} \subset \mathbb{R}^{3}$; voisinage ouvert de $z$ dans $\mathbb{R}^{3}$. Soit $W_{1}=W_{0} \cap \Omega$ et $N=(-\varepsilon, T)_{t} \times$ $\left(W_{0} \cap \partial \Omega\right)_{x}$. On fixe $\rho_{0} \in T^{*} N \backslash 0$ vérifiant $q\left(\rho_{0}\right)=0, \pi\left(\rho_{0}\right)=\left(t_{0}, z\right)$ avec $0 \leqq t_{0}<T$. Ici, $\pi$ est la projection de $T^{*} N$ sur $N$. On note $\partial / \partial n$ la dérivée au long de vecteur normal de $\partial \Omega$. Soit $u={ }^{t}\left(u_{1}, u_{2}, u_{3}\right) \in \mathscr{D}^{\prime}\left((-\varepsilon, T) \times W_{1}\right)$. On dit que $u$ vérifie (A.1) si les conditions suivantes (a) et (b) sont vérifiées.

(a) $L u=0$ dans $(-\varepsilon, T) \times W_{1}$.

(b) Il existe $W_{2} \subset \mathbb{R}^{3}$ voisinage ouvert de $W_{0} \cap \bar{\Omega}$ et $\tilde{u}={ }^{t}\left(\tilde{u}_{1}, \tilde{u}_{2}, \tilde{u}_{3}\right) \in$ $\mathscr{D}^{\prime}\left((-\varepsilon, T) \times W_{2}\right)$ tels que $\left.\tilde{u}\right|_{(-\varepsilon, T) \times W_{1}}=u$ et que $\tilde{u}$ soit analytique dans $(-\varepsilon, 0)$ $\times W_{2}$.

On fixe $m \in \mathbb{R}$ vérifiant $1 \leqq m<3$. Le théorème suivant est notre résultat principal.

Théorème 1. On suppose que (H.1), (H.2) sont vérifiées. Alors, il existe $\omega \subset T^{*} N \backslash 0$ voisinage conique de $\rho_{0}$ et $s_{1}>0$ vérifiant $\exp s_{1} H_{q}\left(\rho_{0}\right) \notin \omega$, tels que, pour tout $u={ }^{t}\left(u_{1}, u_{2}, u_{3}\right) \in \mathscr{D}^{\prime}\left((-\varepsilon, T) \times W_{1}\right)$ vérifiant (A.1) avec $W F_{A}\left(\left.u\right|_{N}\right) \subset \omega$ et tout $s_{0}>0$ vérifiant $s_{0}<s_{1}$ et $\omega \cap\left\{\exp s H_{q}\left(\rho_{0}\right) ; s_{0} \leqq s \leqq s_{1}\right\}=\phi$, on ait (i) et (ii) suivants.

(i) $\left\{\exp s H_{q}\left(\rho_{0}\right) ; s_{0} \leqq s \leqq s_{1}\right\} \cap W F_{G}^{3}\left(\left.(\partial u / \partial n)\right|_{N}\right)=\phi$.

(ii) $\left\{\exp s H_{q}\left(\rho_{0}\right) ; s_{0} \leqq s \leqq s_{1}\right\} \cap W F_{G}^{m}\left(\left.(\partial u / \partial n)\right|_{N}\right)=\phi$

ou $\left\{\exp s H_{q}\left(\rho_{0}\right) ; s_{0} \leqq s \leqq s_{1}\right\} \subset W F_{G}^{m}\left(\left.(\partial u / \partial n)\right|_{N}\right)$.

Remarque. Soit $u={ }^{t}\left(u_{1}, u_{2}, u_{3}\right) \in \mathscr{D}^{\prime}\left((-\varepsilon, T) \times W_{1}\right)$ vérifiant (A.1) et que $W F_{A}\left(\left.u\right|_{N}\right)=\left\{\left(t_{0}, z ; r \eta\right): r>0\right\}$, où $\left(t_{0}, z ; \eta\right)=\rho_{0}$. Alors, la preuve de Théorème 1 montre que l'on a (i) et (ii) mentionnés dans Théorème 1 pour tout $s_{0}$ vérifiant $0<s_{0}<s_{1}$, si $s_{1}$ est suffisamment petit.

Dans la description de Théorème $1, W F_{A}(*)$ est l'ensemble du front d'onde analytique de Hörmander, qui coïncide le spectre singulier de Sato et le support essentiel de Bros-Iagolnitzer. $W F_{G}^{m}(*)$ est l'ensemble du front d'onde Gevrey $m$. On confirme ici seulement la définition de la classe Gevrey $m$ dans $\mathbb{R}^{n}$. On dit qu'une fonction $h$ scalaire en $\mathbb{R}^{n}$ a la régularité Gevrey $m$ près de $y \in \mathbb{R}^{n}$ s'il existe des constantes $B, C>0$ et un voisinage $U$ de $y$ tels que pour tout $\alpha$ et tout $x \in U$, on ait

$$
\left|\left(\partial_{\lambda}^{\alpha} h\right)(x)\right| \leqq C B^{|\alpha|}(\alpha !)^{m}
$$

Pour $u={ }^{t}\left(u_{1}, u_{2}, u_{3}\right)$, on définit $W F_{A} u=\bigcup\left\{W F_{A} u_{j}: j=1,2,3\right\}$ et $W F_{G}^{m} u=$ $\bigcup\left\{W F_{G}^{m} u_{j}: j=1,2,3\right\}$. 
Nous écrivons les travaux qui précèdent Théorème 1. Lebeau [14, Théorème 1] a prouvé Théorème 1 avec une méilleure estimation par rapport à la régularité des solutions lorsque l'équation est celle des ondes. En remplaçant $W F_{G}^{3}$ par $W F$, le front d'onde $C^{\varkappa}$ de Hörmander, Melrose [16] et Taylor [22] (Cf. Hörmander [5]) ont montré Théorème 1-(i), lorsque l'équation est celle des ondes. L'étude de Friedlander-Melrose [4] montre que Théorème 1-(i) est faux si on remplace $W F_{G}^{3}$ par $W F_{G}^{k}$ avec $1 \leqq k<3$ et remplace l'équation élastique par celle des ondes. Voir aussi Sjöstrand [19], Lebeau [13, Théorème 1 dans $\S$ V.2] et [13, Théorème 2 qui étend le résultat de Kataoka]. Théorème 1 est une analogie de Lebeau [14, Théorème 1] pour l'équation élastique isotrope. La singularité des solutions du problème aux limites pour l'équation élastique isotrope a déjà été étudiée par Yamamoto [23] avec la condition de Neumann au bord, dans le cadre de la classe $C^{\propto}$. Cf. Bardos-Masrour-Tatout [1] et Kawashita [7]. La généralisation de Lebeau [14] pour les conditions obliques au bord a été étudiée par Lafitte [8]-[10]. Voir aussi Lascar-Lascar [11].

La preuve de Théorème 1 est essentiellement due aux Lebeau [14] et Stefanov-Vodev [21]. L'idée principale de Lebeau [14] est de construire le paramétrix de l'opérateur au bord sur l'espace conormal de la région Glancing au bord. Cette méthode, appelée la deuxième microlocalisation sur les sousvariétés involutives, provenante de la théorie de Kashiwara-Kawai (C.f. [18]), nous permet de considérer l'algèbre des opérateurs 2-microlocaux de Laurent sur l'espace conormal de la sous variété involutive comme l'algèbre formelle des opérateurs pseudo-différentiels, qui ont 2 paramètres, sur l'espace cotangent. Ces opérateurs pseudo-différentiels sont les opérateurs unilatéraux, qui sont microlocaux jusqu'à l'indice 3 dans classe de Gevrey. Grâce à la deuxième microlocalisation due à Lebeau, on peut inverser l'opérateur au bord en conservant la propriété locale de Gevrey 3. Cela implique la preuve de Théorème 1 lorsque l'équation est celle des ondes. Quant à la deuxième microlocalisation due à Lebeau, Voir [12], [13] et [14, A.63 dans la pages 14891493].

La combinaison de [14] et [21] nous entraîne la preuve de Théorème 1. En effet, $[21, \S 2]$ a montré que l'on peut résoudre le problème aux limites pour l'équation élastique isotrope avec la condition de Dirichlet au bord par la résolution Helmholtzienne. Puisque l'équation élastique est un système, la construction des solutions asymptotiques est beaucoup plus compliquée que celle de l'équation des ondes. Cf. Kawashita [7]. La méthode de [21, §2] nous permet d'éviter de construire directement les solutions asymptotiques dans la région Glancing des ondes longitudinaux et cela indique la preuve de Théorème 1 due à l'argument par [14].

Le plan de cet article est comme ci-dessous. Dans $\S 1$, on définit l'espace de Sjöstrand tangentiel sur lequel on analyse le problème aux limites pour l'équation élastique. Dans $\S 2$, on fait quelques rappels sur l'équation des 
ondes, qui joueront le rôle principal pour la preuve de Théorème 1. Dans $\S 3$, on décrit notre résultat, qui implique Théorème 1, dans l'espace de Sjöstrand. Dans $\S 4$, on étudie l'algèbre des opérateurs unilatéraux et son action sur des fonctions dans l'espace de Sjöstrand. Dans $\S 5$, on prouve Théorème 1 par la combinaison de [14] et [21]. Dans $\S 6$, on fait quelques rappels sur Gevrey holomorphie partielle, qui sont utilisés dans la preuve de Théorème 1 .

\section{§1. L'espace de Sjöstrand pour les Problèmes aux Limites}

Soit $X \subset \mathbb{C}^{3}$ ouvert et $\varphi$ une fonction scalaire et continue sur $X$ à valeur réelle.

Définition 1.1. L'espace de Sjöstrand $H_{\varphi}(X)$ est l'ensemble des fonctions dont les valeurs sont vecteurs; $f(x, \lambda)={ }^{t}\left(f_{1}, f_{2}, f_{3}\right)(x, \lambda), x \in X, \lambda>0$, holomorphes en $x \in X$ telles que pour tout $K \Subset X$ compact et tout $\varepsilon>0$ il existe $C>0$ tel que pour tout $x \in K$, tout $\lambda \geqq 1$ on ait

$$
|f(x, \lambda)| \leqq C \exp (\lambda(\varphi(x)+\varepsilon))
$$

ò̀ $|f(x, \lambda)|=\left(\sum_{j=1}^{3}\left|f_{j}(x, \lambda)\right|^{2}\right)^{1 / 2}$.

Définition 1.2. Soit $f \in H_{\varphi}(X)$ et $y \in X$. On dit que $y \notin S S_{\varphi}^{X}(f)$ s'il existe un voisinage ouvert $W \subset X$ de $y$ et $c>0$ tels que $f \in H_{\varphi-c}(W)$. L'ensemble $S S_{\varphi}^{X}(f)$ est appelé le spectre analytique de $f$ dans $H_{\varphi}(X)$.

Définition 1.3. Soit $g \in H_{\varphi}(X)$. On dit que $g$ est à croissance dans $X$ s'il existe $M>0$ tel que, pour tout $x \in X$ et tout $\lambda \geqq 2$, on ait

$$
|g(x, \lambda)| \leqq \lambda^{M} \exp (\lambda \varphi(x)) \text {. }
$$

Définition 1.4. Soit $\rho>0 . H_{\varphi}^{+, \rho}(X)$ est l'ensemble des fonctions $f\left(x_{4}, x, \lambda\right)={ }^{t}\left(f_{1}, f_{2}, f_{3}\right)\left(x_{4}, x, \lambda\right)$; holomorphe en $x \in X$ et en $x_{4} \in U$ avec un voisinage ouvert complex $U$ de l'intervalle fermé $[0, \rho]$ dans $\mathbb{C}$, définies pour $\lambda>0$, vérifiant les conditions suivantes:

(C.1) Pour tout $K \Subset X$ compact, il existe $C>0$ telle que, pour tout $x \in K$, $x_{4} \in U, \lambda \geqq 1$, on ait

$$
\left|f\left(x_{4}, x, \lambda\right)\right| \leqq \exp (C \lambda) .
$$

(C.2) Pour tout $K \Subset X$ compact, tout $\varepsilon>0$ et tout $m \in \mathbb{N}$, il existe $C>0$ telle que, pour tout $x_{4} \in[0, \rho)$, tout $x \in K$ et tout $\lambda \geqq 1$, on ait

$$
\left|\left(\partial / \partial x_{4}\right)^{m} f\left(x_{4}, x, \lambda\right)\right| \leqq C \exp (\lambda(\varphi(x)+\varepsilon)) .
$$

Ici, $\mathbf{N}=\{1,2,3 \ldots\}$.

Définition 1.5. Pour $f \in H_{\varphi}^{+, \rho}(X)$, son spectre analytique, noté par $S S_{\partial, \varphi}^{X . \rho}(f)$ est le sous-ensemble de $X \cup\left(T^{*}(0, \rho) \times X\right)$ défini comme ci-dessous: 
(C.3) $X \ni y \notin S S_{\imath, \varphi}^{X, \rho}(f)$ s'il existe un voisinage $W$ de $y$ dans $X, r>0, \varepsilon>0$ tels que, pour tout $x \in W$, tout $x_{4} \in \mathbf{C} ;\left|x_{4}\right|<r$ et tout $\lambda \geqq 1$, on ait

$$
\left|f\left(x_{4}, x, \lambda\right)\right| \leqq(1 / \varepsilon) \exp (\lambda(\varphi(x)-\varepsilon)) .
$$

(C.4) $\left(T^{*}(0, \rho) \times X\right) \ni\left(y_{4}, \eta_{4}, ; y\right) \notin S S_{\partial, \varphi}^{X, \rho}(f)$ s'il existe un voisinage $W$ de $y$ dans $X, r>0, \varepsilon>0$ et $\delta>0$ vérifiant $\delta<\inf \left\{y_{4}, \rho-y_{4}\right\}$ tels que l'on ait

$$
\begin{array}{r}
\left|\int_{y_{4}-\delta}^{y_{4}+\delta} \exp \left(-(\lambda / 2)\left(z_{4}-x_{4}\right)^{2}\right) f\left(x_{4}, x, \lambda\right) d x_{4}\right| \\
\leqq(1 / \varepsilon) \exp \left(\lambda\left(\varphi(x)+\left|\operatorname{Im} z_{4}\right|^{2} / 2-\varepsilon\right)\right)
\end{array}
$$

pour tout $z_{4} \in \mathbf{C}$ vérifiant $\left|z_{4}-\left(y_{4}-i \eta_{4}\right)\right|<r$, tout $x \in W$ et tout $\lambda \geqq 1$.

Remarque 1.6. Quant à la relation entre la singularité analytique dans $T^{*} \mathbf{R}^{n}$ et le spectre analytique dans l'espace de Sjöstrand, on renvoie à Sjöstrand [20].

Remarque 1.7. Quant aux notations $S S_{\varphi}^{X}, S S_{\partial, \varphi}^{X, \rho}$, on n'écrira pas les indices $X, \rho, \varphi$ etc. lorsqu'ils ne sont pas importants.

Dans cet article, on utilisera les notatios suivantes. Soit $\psi$ une fonction scalaire et dérivable sur $X$ à valeur réelle et $x \in X$. On définit $j_{\psi}(x) \in T^{*} X$ par $j_{\psi}(x)=(x,-2 i(\partial \psi / \partial x)(x))$. Pour $W \subset X$, on définit $\Lambda_{\psi}(W)$ par $\Lambda_{\psi}(W)=$ $\left\{x \in W: j_{\psi}(x)\right\}$. Pour $x \in X$, on note $x=\left(x_{1}, x^{\prime}\right)$ avec $x_{1} \in \mathbf{C}$ et $x^{\prime} \in \mathbf{C}^{2}$. On définit $\varphi_{0}(x)$ par $\varphi_{0}(x)=|\operatorname{Im} x|^{2} / 2$. On note $I_{0}$ le $3 \times 3$ matrice d'unité et $\mathbf{N}=$ $\{1,2,3 \ldots\}$.

\section{§2. Rappels sur l'Équation des Ondes}

Dans cette section, nous faisons quelques rappels sur l'équation des ondes, qui joueront le rôle principal pour la preuve de Théorème 1 . On conserve ici les notations dans $\S 0$ et $\S 1$.

Soit $\square_{J}=\left((\partial / \partial t)^{2}-c_{J}^{2} \Delta\right) I_{0}$ dans $\mathbf{R}_{t} \times \mathbf{R}_{x}^{3}$, où $c_{1}=\left(\lambda_{0}+2 \mu_{0}\right)^{1 / 2}, c_{2}=\mu_{0}^{1 / 2}$. On suppose que (H.1) et (H.2) sont vérifiées. Selon Bardos-Lebeau-Rauch $[1, \S 5]$ ou Lafitte $[8, \S 5]$, il existe une coordonné $\left(y^{\prime \prime}, y_{3}\right) ; y^{\prime \prime} \in \mathbf{R}^{2}, y_{3} \in \mathbf{R}$ et une fonction scalaire et analytique $\beta\left(y^{\prime \prime}, y_{3}\right)$ près de $z \in \partial \Omega$ vérifiant $\beta=1$ sur $\left\{y_{3}=0\right\}$ telles que, comme l'opérateur différentiel, on ait

$$
\beta^{-1} \Delta \beta=\left(\partial / \partial y_{3}\right)^{2}+q\left(y^{\prime \prime}, y_{3},-i \partial / \partial y^{\prime \prime}\right),
$$

où $q$ est l'opérateur différentiel scalaire avec l'ordre 2 dont la partie principale est réelle. Ici, $\left\{y_{3}>0\right\}$ correspend localement à $\Omega$. D'après (2.1), on a

$$
c_{j}^{-2} \beta^{-1} \square_{\jmath} \beta=-\left(\partial / \partial y_{3}\right)^{2}+\left(-q\left(y^{\prime \prime}, y_{3},-i \partial / \partial y^{\prime \prime}\right)+c_{j}^{-2}(\partial / \partial t)^{2}\right) .
$$


Remarque. La notation $q \grave{a}(2.1)$ et (2.2) est différente de $q \grave{a} \S 0$.

Prenons la transformation FBI tangentielle associée à la transformation canonique

$$
\chi_{1}:\left(T^{*} N, \omega_{0}\right) \rightarrow(X,-2 i \bar{\partial} \partial \varphi)
$$

où $X \subset \mathbf{C}^{3}$ est ouvert, $\varphi$ est la fonction scalaire qui est strictement pluri sousharmonique sur $X, \omega_{0}$ est la 2 forme canonique de $T^{*} N$ et $N=(-\varepsilon, T) \times$ $\left(W_{0} \cap \partial \Omega\right)$. Selon $[1, \S 5]$, cette transformation FBI entraîne la transformation;

$$
\begin{gathered}
T^{*} N \ni \rho_{0} \rightarrow x_{0} \in X \\
\left(c_{J}^{-2} \beta^{-1} \square_{J} \beta\right) I_{0} \rightarrow \lambda^{2} P_{J}, \quad j=1,2
\end{gathered}
$$

où

$$
\begin{aligned}
\left(P_{j} f\right)\left(x_{4}, x, \lambda\right) & \\
= & \lambda^{-2}\left(\partial / \partial x_{4}\right)^{2} f\left(x_{4}, x, \lambda\right) \\
& +(2 \pi / \lambda)^{3} \int_{\Sigma_{0}} \exp (i \lambda(x-y) \xi)\left(\ell^{(J)}\left(x_{4}, x, \xi, \lambda\right) I_{0}\right) f\left(x_{4}, y, \lambda\right) d y \wedge d \xi
\end{aligned}
$$

$\Sigma_{0}:$ le contour d'intégration; $\xi=-2 i(\partial \varphi / \partial x)(x)+i C(\bar{x}-\bar{y}), \quad|x-y| \leqq r$ avec $C \geqq 1$ suffisamment grand, $r C$ suffisamment petit,

$f \in H_{\varphi}^{+, \rho}(X)$ avec $\rho>0$ suffisamment petit,

$\ell^{(J)}$ : symbole (scalaire) analytique classique de degré 0 , c.-á-d. il existe une suite $\left\{\ell_{k}^{(J)}\left(x_{4}, x, \xi\right)\right\}_{k=0}^{\infty}$ de fonction holomorphe sur $\Omega_{0} \subset \mathbb{C} \times T^{*} X$ voisinage complex ouvert de $\left[0, \rho_{\rfloor}^{\top} \times\left\{j_{\varphi}\left(x_{0}\right)\right\}\right.$ et des constantes $A$ et $B$ tels que pour tout $M$ et tout $\lambda \geqq 1$ on ait

$$
\begin{aligned}
& \sup \left\{\left|\ell^{(j)}\left(x_{4}, x, \xi, \lambda\right)-\sum_{k=0}^{M} \ell_{k}^{(\jmath)}\left(x_{4}, x, \xi\right) \lambda^{-k}\right|:\left(x_{4}, x, \xi\right) \in \Omega_{0}\right\} \\
& \leqq A B^{M} M ! \lambda^{-M-1}
\end{aligned}
$$

La région elliptique $E^{J}$, hyperbolique $H^{J}$, Glancing $G^{J}$ dans $X$ pour chaque $P_{j} ; j=1,2$ est défini par

$$
\begin{aligned}
& E^{J}=\pi\left\{\Lambda_{\varphi}(X) \cap\left\{r_{0}^{(J)}<0\right\}\right\}, \quad H^{J}=\pi\left\{\Lambda_{\varphi}(X) \cap\left\{r_{0}^{(J)}>0\right\}\right\}, \\
& G^{J}=\pi\left\{\Lambda_{\varphi}(X) \cap\left\{r_{0}^{(J)}=0\right\}\right\},
\end{aligned}
$$

où $\pi$ est la projection de $T^{*} X$ sur $X, r_{0}^{(J)}(x, \xi)=\ell_{0}^{(J)}(0, x, \xi)$. Notons que l'on a $x_{0} \in G^{1}$. 
Comme Lebeau $[14, \S 1]$, on peut supposer que

$$
\begin{array}{ll}
\varphi(x)=\varphi_{0}(x)=|\operatorname{Im} x|^{2} / 2, & E^{1}=\left\{z \in X: \operatorname{Im} z_{1}>0\right\}, \\
H^{1}=\left\{z \in X: \operatorname{Im} z_{1}<0\right\}, & G^{1}=\left\{z \in X: \operatorname{Im} z_{1}=0\right\}, \\
x_{0}=\left(0, x_{0}^{\prime}\right) \text { avec } x_{0}^{\prime} \in \mathbf{C}^{2}, & \operatorname{Re} x_{0}^{\prime}=0 .
\end{array}
$$

Définition 2.1. Soit $(y, \eta) \in \Lambda_{\varphi_{0}}(X)$. Les flots sortants de $(y, \eta)$, notés $F^{J}(y, \eta), j=1,2$ sont les sous-ensembles de $\Lambda_{\varphi_{0}}(X) \cup\left(T^{*} \mathbf{R}_{+} \times \Lambda_{\varphi_{0}}(X)\right)$ définis comme ci-dessous.

Lorsque $(y, \eta) \in \Lambda_{\varphi_{0}}\left(E^{J}\right), F^{J}(y, \eta)=\{(y, \eta)\}$.

Lorsque $(y, \eta) \in \Lambda_{\varphi_{0}}\left(G^{\prime} \cup H^{J}\right), F^{J}(y, \eta)=\{(y, \eta)\} \cup\left\{\exp s H_{p^{(\jmath)}}\left(y_{4}, \eta_{4} ; y, \eta\right)\right.$ :

$s>0\}$ avec $p^{(j)}=-\xi_{4}^{2}+\ell_{0}^{(J)}\left(x_{4}, x, \xi\right), y_{4}=0, \eta_{4}=\phi_{j}(y, \eta)$,

$\phi_{J}(y, \eta)$ : la solution de $\phi_{J}(y, \eta)^{2}=r_{0}^{(j)}(y, \eta), \phi_{j}(y, \eta) \leqq 0$,

$H_{p^{(1)}}$ : le champ hamiltonien de $p^{(J)}$.

Remarque. $\ell_{0}^{(J)}\left(x_{4}, x, \xi\right)$ est réel si $x_{4} \in \mathbf{R}_{+}$et $(x, \xi) \in \Lambda_{\varphi_{0}}(X)$.

Soit $g \in H_{\varphi_{0}}(X)$ avec $S S_{\varphi_{0}}^{X}(g)$ suffisamment petit dans $X, \rho>0$ suffisamment petit, $j=1$ ou 2 et $\tilde{\pi}$ la projection de $T^{*} X \cup\left(T^{*} \mathbf{R}_{+} \times T^{*} X\right)$ sur $X \cup$ $\left(T^{*} \mathbf{R}_{+} \times X\right)$.

Définition 2.2. On dit que $f \in H_{\varphi_{0}}^{+, \rho}(X)$ est la solution sortante de l'équation

$$
\begin{aligned}
& \left\{\begin{array}{l}
P_{J} w=0 \\
\left.w\right|_{x_{4}=0}=g
\end{array}\right. \\
& \text { si }\left\{\begin{array}{l}
S S_{\partial, \varphi_{0}}^{W, \rho}\left(P_{j} f\right)=\phi, \\
f(0, x, \lambda)=g(x, \lambda), \\
S S_{\partial, \varphi_{0}}^{W, \rho}(f) \subset \tilde{\pi} \circ F^{J}\left(\Gamma_{j}\right),
\end{array}\right.
\end{aligned}
$$

ò̀ $\quad \Gamma_{J}=\Lambda_{\varphi_{0}}\left(S S_{\varphi_{0}}^{X}(g)\right) \cup\left\{\exp s H_{r_{0}^{(J)}}\left(\Lambda_{\varphi_{0}}\left(S S_{\varphi_{0}}^{X}(g) \cap G^{j}\right)\right): s>0\right\} \quad$ et $\quad W \quad$ est un voisinage ouvert de $S S_{\varphi_{0}}^{X}(g)$ dans $X$.

Définition 2.3. L'opérateur $B_{m}^{[j]}:\left\{g \in H_{\varphi_{0}}(X): g\right.$ est à croissance dans $\left.X\right\}$ $\rightarrow H_{\varphi_{0}}\left(X_{0}\right)$ avec $j \in \mathbf{N} \cup\{0\}, m=1,2, x_{0} \in X_{0} \Subset X, X_{0}$ suffisamment petit, est défini par

$$
B_{m}^{[J]}=\left.\left(\left(\partial / \partial x_{4}\right)^{J} R_{m} Q_{m} g\right)\right|_{x_{4}=0} \quad \text { avec }
$$

$Q_{m} g$ : la solution sortante de (2.6) dans lequel $j$ est remplacé par $m$, $R_{1}$ : réalisation dans $H_{\varphi_{0}}^{+, \rho}(X)$ de l'opérateur différentiel $\beta^{-1} \operatorname{grad} \operatorname{div} \beta$ dans $\mathbf{R}_{r}^{3}$, 
$R_{2}$ : réalisation dans $H_{\varphi_{0}}^{+, \rho}(X)$ de l'opérateur différentiel $-\beta^{-1} \operatorname{rot} \operatorname{rot} \beta$ dans $\mathbf{R}_{\mathrm{x}}^{3}$.

Ici, ces réalisations sont faites par la même transformation FBI tangentielle associée à (2.3) dans §2. La notation "rot" signifie la rotation de vecteur.

Soit $\tilde{x} \in H^{j}, j=1$ ou 2 . Selon la propriété de la solution de l'équation des ondes dans la région hyperbolique, $B_{m}^{[j]}$ est opérateur pseudo-différentiel à $\tilde{x}$, c.-à-d. il existe $b^{J, m}(x, \xi, \lambda)$ le symbol analytique classique formel de degré 0 à $\tilde{x}$, dont la valeur est $3 \times 3$ matrice, avec la somme formelle

$$
b^{J, m}(x, \xi, \lambda)=\sum_{k=0}^{\infty} b_{k}^{\jmath, m}(x, \xi) \lambda^{-k},
$$

telle que

$$
\begin{aligned}
\lambda^{-6}\left(B_{m}^{[j]} g\right)(x, \lambda)= & (\lambda / 2 \pi)^{3} \sum_{0 \leqq k \leqq \lambda / M} \int_{\Sigma_{0}} \exp (i \lambda(x-y) \xi) \\
& \times \lambda^{-k} b_{k}^{j, m}(x, \xi) g(y, \lambda) d y \wedge d \xi
\end{aligned}
$$

$\Sigma_{0}$ : contour d'intégration, $\xi=-\operatorname{Im} x+i C(\bar{x}-\bar{y}), \quad|x-y|<r, \quad C \gg 1, \quad 0<$ $r C \ll 1$ et $M \gg 1$. Voir Sjöstrand [19] et Stefanov-Vodev [21].

On utilisera dans $\S 5$ l'opérateur $B_{m}^{[J]}$ afin de réduire l'analyse de l'équation élastique à celle de l'équation des ondes.

\section{§3. La Description du Résultat dans l'Espace de Sjöstrand}

On conserve les notations dans $\S 0-\S 2$. Soit $M$ la réalisation dans $H_{\varphi_{0}}^{+, \rho}(X)$ de l'opérateur différentiel $-(\partial / \partial t)^{2}+\beta^{-1} A\left(\partial_{x}\right) \beta$ dans $\mathbb{R}_{t} \times \mathbb{R}_{1}^{3}$, faite par la même transformation FBI tangentielle associée à (2.3), que l'on a utilisé dans $\S 2$. Alors, $M$ est la $3 \times 3$ matrice dont chaque élément est l'opérateur différentiel par rapport à $x_{4}$ et celui pseudo-différentiel par rapport à $x \in X$.

Définition 3.1. Soit $g \in H_{\varphi_{0}}(X)$ avec $S_{\varphi_{0}}^{X}(g)$ suffisamment petit dans $X$. On dit que $f \in H_{\varphi_{0}}^{+, \rho}(X)$ est solution sortante de

$$
\begin{aligned}
& \left\{\begin{array}{l}
M w=0 \\
\left.w\right|_{\lambda_{4}=0}=g
\end{array}\right. \\
& \text { si }\left\{\begin{array}{l}
S S_{\hat{\partial}, \varphi_{0}}^{W, \rho}(M f)=\phi, \\
f(0, x, \lambda)=g(x, \lambda) \\
S S_{\partial, \varphi_{0}}^{W, \rho}(f) \subset \tilde{\pi}\left(F^{1}(A) \cup F^{2}(A)\right)
\end{array}\right.
\end{aligned}
$$


où

$$
\begin{aligned}
& A=\Lambda_{\varphi_{0}}\left(S S_{\varphi_{0}}(g)\right) \cup K_{1} \cup K_{2}, \\
& K_{J}=\left\{\exp s H_{r_{0}^{(\jmath)}}\left(\Lambda_{\varphi_{0}}\left(S S_{\varphi_{0}}^{X}(g) \cap G^{J}\right)\right): s>0\right\}, \quad j=1,2 \\
& W: \text { un voisinage ouvert de } S S_{\varphi_{0}}^{X}(g), \\
& \tilde{\pi}: \text { la projection de } T^{*} X \cup\left(T^{*} \mathbf{R}_{+} \times T^{*} X\right) \text { sur } X \cup\left(T^{*} \mathbf{R}_{+} \times X\right) .
\end{aligned}
$$

Soit $-1 \ll a_{1}<\varepsilon_{0}<0<\varepsilon_{1}<b_{1} \ll 1,0<\varepsilon_{J} \ll 1, j=2,3$ et un voisinage ouvert $\omega_{1} \subset X$ de $x_{0}$ vérifiant $\omega_{1} \subset\left\{x \in X: \varepsilon_{0}<\operatorname{Re} x_{1}<\varepsilon_{1},\left|\operatorname{Im} x_{1}\right|<\varepsilon_{2}\right.$, $\left.\left|x^{\prime}-x_{0}^{\prime}\right|<\varepsilon_{3}\right\}$. Notre but est de prouver le théorème suivant, qui implique Théorème 1 .

Théorème 3.2. Soit $g \in H_{\varphi_{0}}(X)$, qui est à croissance, vérifiant $S S_{\varphi_{0}}(g) \subset \omega_{1}$. Soit $f \in H_{\varphi_{0}}^{+, \rho}(X)$ la solution sortante de (3.1). Alors, pour tout $v \in G^{1}$ vérifiant $\varepsilon_{1}<\operatorname{Re} v_{1}<b_{1}$, il existe un voisinage $\omega_{2} \subset X$ de $v, C>0, \delta>0$ tels que pour tout $x \in \omega_{2}, \lambda \geqq 1$, on ait

$$
\left|\frac{\partial f}{\partial x_{4}}(0, x, \lambda)\right| \leqq C \exp \left(\frac{\lambda}{2}\left|\operatorname{Im} x^{\prime}\right|^{2}-\delta \lambda^{1 / 3}\right) .
$$

La preuve de Théorème 3.2 sera donnée dans $\S 5$.

\section{§4. Opérateurs Unilatéraux}

On étudie ici l'algèbre des opérateurs unilatéraux et son action sur des fonctions dans l'espase de Sjöstrand. L'argument est essentiellement pareil à Lebeau $[14, \S 4]$.

\section{4-1. Algèbre des Opérateur Unilatéraux}

Dans $T^{*} \mathbf{C}^{3}$ muni du systéme de coordonées $(x, \xi): x=\left(x_{1}, x_{2}, x_{3}\right)=$ $\left(x_{1}, x^{\prime}\right), \xi=\left(\xi_{1}, \xi_{2}, \xi_{3}\right)=\left(\xi_{1}, \xi^{\prime}\right)$, on définit $V \subset T^{*} \mathbf{C}^{3}$ par

$$
V=\left\{(x, \xi) \in T^{*} \mathbf{C}^{3}: \xi_{1}=0\right\}
$$

et $V_{0} \subset \mathbf{C}^{3}$ par $V_{0}=\pi\left(V \cap \Lambda_{\varphi_{0}}\left(\mathbf{C}^{3}\right)\right)$, où $\varphi_{0}(x)=|\operatorname{Im} x|^{2} / 2$ et $\pi$ est la projection de $T^{*} \mathbf{C}^{3}$ sur $\mathbf{C}^{3}$. Alors, on a $V_{0}=\left\{x \in \mathbf{C}^{3}: \operatorname{Im} x_{1}=0\right\}$.

Soit $m \in V$ avec $m=\left(z_{0} ; 0, \xi_{0}^{\prime}\right), z_{0} \in \mathbf{C}^{3}, \xi_{0}^{\prime} \in \mathbf{C}^{2}$. Pour $s>0$ et $\delta>0$, on définit $\Omega(\lambda, s, \delta, \gamma)$ par

$$
\begin{aligned}
\Omega(\lambda, s, \delta, \gamma)=\{ & (x, \xi) \in T^{*} \mathbf{C}^{3}:\left|x-z_{0}\right|<s,\left|\xi^{\prime}-\xi_{0}^{\prime}\right|<s,\left|\xi_{1}\right|<s \\
& \left.\operatorname{Im} \xi_{1}-\gamma \lambda^{-2 / 3}<\delta\left|\operatorname{Re} \xi_{1}\right|\right\} .
\end{aligned}
$$


Lorsque $f(x, \xi, \lambda)$ est une fonction à valeur $3 \times 3$ matrice définie sur $\Omega(\lambda, s, \delta, \gamma)$, pour $\lambda \geqq 1$, holomorphe en $(x, \xi)$, on pose, pour tout entier $n \geqq 0$,

$$
|f|_{s, \delta, n}(\lambda)=\sup \left\{\left|\left(\xi_{1}-i \gamma \lambda^{-2 / 3}\right)^{n} f(x, \xi, \lambda)\right|:(x, \xi) \in \Omega(\lambda, s, \delta, \gamma)\right\} .
$$

Ici, $|a|=\left(\sum_{k, J=1}^{3}\left|a_{k, j}\right|^{2}\right)^{1 / 2}$ lorsque $a$ est la $3 \times 3$ matrice dont chaque élément est $a_{k, j}$. On note $B_{s, \delta, n}$ l'ensemble des $f$ tels que $|f|_{s, \delta, n}(\lambda)$ soit fini et borné comme fonction de $\lambda$.

Définition 4.1.1. $\mathscr{E}_{V, m, \gamma}^{+}$est l'ensemble des séries formelles

$$
p=\sum_{k=0}^{\infty} p_{k}(x, \xi, \lambda)(i T)^{-k}
$$

telles qu'il existe $s, \delta, A, B>0$ vérifiant $p_{k} \in B_{s, \delta, k}$ et

$$
\left|p_{k}\right|_{s, \delta, k} \leqq A B^{k} k !
$$

pour tout $k \geqq 0$. On appelle $p_{0}$ la partie principale de $p$.

Pour $p, q \in \mathscr{E}_{V, m, \gamma}^{+}$, on définit $b=p * q$ par

$$
b=\sum_{n=0}^{\infty} b_{n}(x, \xi, \lambda)(i T)^{-n} \quad \text { avec } \quad b_{n}=\sum_{|\alpha|+J+k=n}(\alpha !)^{-1} \partial_{\xi}^{\alpha} p_{k} \partial_{x}^{\alpha} q_{\jmath} .
$$

Selon Lebeau [14, Proposition 4.1.1 dans $\S 4]$ et sa démonstration, on peut savoir que $\left(\mathscr{E}_{V, m, \gamma}^{+}, *,+\right)$ est anneau. Voir aussi Lafitte [8, Proposition 3 dans $\S 3]$.

Définition 4.1.2. On dit que $q \in \mathscr{E}_{V, m . \gamma}^{+}$est elliptique s'il existe $s, \delta, M>0$, qui sont indépendants de $\lambda$, tels que

$$
1 / M \leqq\left|\operatorname{det} q_{0}(x, \xi, \lambda)\right| \leqq M \quad \text { pour } \quad(x, \xi) \in \Omega(\lambda, s, \delta, \gamma),
$$

où $q_{0}$ est la partie principale de $q$.

Selon Lebeau [14, Proposition 4.1.3] et sa démonstration, on obtient la proposition suivante:

Proposition 4.1.3. (Lebeau [14]) Soit $q \in \mathscr{E}_{V, m, \gamma}^{+}$elliptique. Alors, il existe un unique $p \in \mathscr{E}_{V, m, \gamma}^{+}$tel que $q * p=p * q=1$.

\section{§4-2. Actions des Opérateurs}

Soit $m=\left(z_{0} ; 0, \xi_{0}^{\prime}\right) \in V$ mentionné dans $\S 4-1$. Dans cette section, on suppose que $m \in \Lambda_{\varphi_{0}}\left(V_{0}\right)$. Alors, on a $z_{0} \in V_{0}$ et $\xi_{0}^{\prime}=-\operatorname{Im} z_{0}^{\prime}$. Ici, $z_{0}=$ $\left(z_{0,1}, z_{0}^{\prime}\right) \in \mathbb{C} \times \mathbb{C}^{2}$. On suppose aussi que $z_{0,1}=0$. 
On définit $\Omega_{0} \subset \mathbf{C}$ par

$$
\Omega_{0}=\left\{x \in \mathbf{C}: a_{0}<\operatorname{Re} x<b_{0},|\operatorname{Im} x|<c_{0}\right\}
$$

avec $-1 \ll a_{0}<0<b_{0} \ll 1,0<c_{0} \ll 1$.

Soit $w \in H_{\varphi_{0}}\left(\bar{\Omega}_{0} \times U\right)$ avec un voisinage ouvert $U$ de $z_{0}^{\prime}$ dans $\mathbf{C}^{2}$. Ici, $H_{\varphi_{0}}\left(\bar{\Omega}_{0} \times U\right)$ est l'ensemble de $v \in H_{\varphi_{0}}\left(\Omega_{0} \times U\right)$ vérifiant $\sup \left\{\exp \left(-\lambda\left(\varphi_{0}(y)+\right.\right.\right.$ $\left.\varepsilon))|v(y, \lambda)|: y \in \Omega_{0} \times K_{0}, \lambda \geqq 1\right\}<\infty$ pour tout $\varepsilon>0$ et tout $K_{0} \Subset U$ compact. On définit alors la tronquature $T_{0}$ par

$$
\begin{aligned}
\left(T_{0} w\right)(x, \lambda)= & \frac{\lambda}{2 \pi} \int_{\Omega_{0}} \exp \left(-\frac{\lambda}{4}\left(x_{1}-\bar{y}_{1}\right)^{2}-\lambda\left|\operatorname{Im} y_{1}\right|^{2}\right) \\
& \times w\left(y_{1}, x^{\prime}, \lambda\right) d\left(\operatorname{Re} y_{1}\right) d\left(\operatorname{Im} y_{1}\right) .
\end{aligned}
$$

Comme Lebeau $[14, \S 4.2]$, on a $\left(x_{1}, x^{\prime}\right) \notin S S_{\varphi_{0}}\left(T_{0} w-w\right)$ si $x_{1} \in \Omega_{0}$ et $\left(x_{1}, x^{\prime}\right) \notin$ $S S_{\varphi_{0}}\left(T_{0} w\right)$ si $x_{1} \notin \bar{\Omega}_{0}$. Voir aussi Lebeau [14, Estimation 4.2.3 dans la page 1469].

Soit $\gamma>0$. On définit $D_{\gamma}^{-k} w: k=0,1,2 \ldots$ par

$$
\left\{\begin{aligned}
D_{\gamma^{0}}^{0} w=w & \\
\left(D_{\gamma^{\prime}}^{-k} w\right)\left(x_{1}, x^{\prime}, \lambda\right)= & \int_{a}^{\lambda_{1}} \exp \left(-\gamma \lambda^{1 / 3}\left(x_{1}-s\right)\right) \\
& \times \frac{\left(x_{1}-s\right)^{k-1}}{(k-1) !} w\left(s, x^{\prime}, \lambda\right) d s, \quad k \geqq 1 .
\end{aligned}\right.
$$

avec $a=a_{0}-d, d>0$ suffisamment petit. Selon Lebeau [14, Estimation 4.2.19 dans la page 1471], on a

$$
\begin{aligned}
\mid \exp ( & \left.-\lambda \varphi_{0}(x)\right) D_{\gamma}^{-k}\left(T_{0} w\right)(x, \lambda) \mid \\
\quad \leqq & (\lambda / 2 \pi)\left(\Delta_{0}^{k} / k !\right) \sup \left\{\left|\exp \left(-\lambda \varphi_{0}(y)\right) w(y, \lambda)\right|: y \in \Omega_{0}, y^{\prime} \in U\right\}
\end{aligned}
$$

pour tout $x=\left(x_{1}, x^{\prime}\right)$ avec $x_{1} \in \Omega_{0}, x^{\prime}$ près de 0 et tout $\lambda \geqq 1$. Ici,

$$
\Delta_{0}=\left(c_{0}^{2}+\left(b_{0}-a\right)^{2}\right)^{1 / 2} .
$$

Soit $p \in \mathscr{E}_{V, m, \gamma}^{+}$avec

$$
p=\sum_{k=0}^{\infty} p_{k}(x, \xi, \lambda)(i T)^{-k}
$$

On suppose que

$$
B \Delta_{0}<1
$$


avec $B$ à (4.1.4) et $\Delta_{0}$ à (4.2.6). On définit $O p_{2}^{\gamma}(p)$ par

$$
O p_{2}^{\gamma}(p) w=\sum_{k=0}^{\infty} O p_{3}^{\gamma}\left(\tilde{p}_{k}\right)\left(D_{\gamma}^{-k} T_{0} w\right)
$$

où

$$
\begin{gathered}
\int_{\xi_{1}^{-}}^{\xi_{1}^{+}} d \xi_{1} \exp (i \lambda(x-y) \xi) \tilde{p}_{k}(x, \xi, \lambda)\left(T_{0} v\right)(y, \lambda), \\
\Sigma^{\prime}: \text { contour d'intégration, } \quad \xi^{\prime}=-\operatorname{Im} x^{\prime}+i C\left(\bar{x}^{\prime}-\bar{y}^{\prime}\right), \\
\left|x^{\prime}-y^{\prime}\right|<r, \quad C \gg 1, \quad 0<r C \ll 1, \quad d>0, \quad b=b_{0}+d, \\
\xi_{1}^{ \pm} \in \mathbf{R}, \quad \xi_{1}^{+}=-\xi_{1}^{-}>0, \quad\left|\xi_{1}^{ \pm}\right|>c_{0}, \quad d^{2}>4\left|\xi_{1}^{ \pm}\right| c_{0} .
\end{gathered}
$$

Selon Lebeau [14, Estimation 4.2.5 dans la page 1469] et l'hypothèse (4.2.8), la somme du membre droit de (4.2.9) converge lorsque $w \in H_{\varphi_{0}}\left(\bar{\Omega}_{0} \times U\right)$. En plus, on obtient

$$
O p_{2}^{\gamma}(p) w \in H_{\varphi_{0}}\left(\bar{\Omega}_{0} \times U^{\prime}\right)
$$

avec $z_{0}^{\prime} \in U^{\prime} \Subset U, U^{\prime}$ suffisamment petit, lorsque $w \in H_{\varphi_{0}}\left(\bar{\Omega}_{0} \times U\right)$.

Soit $q$ un symbole analytique classique formel à $m$, dont la valeur est $3 \times 3$ matrice, avec la somme formelle

$$
q=\sum_{k=0}^{\infty} q_{k}(x, \xi)(i \lambda)^{-k} .
$$

On définit $\mu(q)$ par la somme formelle

$$
\mu(q)=\sum_{k=0}^{\infty} q_{k}(x, \xi)(i T)^{-k} .
$$

Alors, il existe $\gamma>0$ tel que l'on ait $\mu(q) \in \mathscr{E}_{V, m, \gamma}^{+}$. Voir Définition 4.1.1 et Lebeau [13, III.2]. On définit $\widetilde{O p_{2}^{\gamma}}(q)$ par

$$
\widetilde{O p}_{2}^{\gamma}(q)=O p_{2}^{\gamma}(\mu(q)) \text {. }
$$

Soit $p \in \mathscr{E}_{V, m, \gamma}^{+}$avec la somme formelle (4.2.7) et $z_{1} \in \mathbb{C}^{3}$ près de $z_{0}$ vérifiant $z_{1} \notin$ $V_{0}$. On définit $v(p)$ par la somme formelle

$$
v(p)=\sum_{k=0}^{\infty} p_{k}(x, \xi, \lambda)(i \lambda)^{-k}
$$


On définit $\widetilde{O p_{1}}(p)$, la quantification de $p$ près de $j_{\varphi_{0}}\left(z_{1}\right) \in \Lambda_{\varphi_{0}}\left(\mathbf{C}^{3}\right)$, par

$$
\widetilde{O p}_{1}(p)=O p_{1}(v(p)),
$$

où $O p_{1}(v(p))$ est la quantification ordinaire de $v(p)$, c.-à-d.

$$
\begin{aligned}
& \left(O p_{1}(v(p)) v\right)(x, \lambda)=\sum_{0 \leqq k \leqq \lambda / M}(\lambda / 2 \pi)^{4} \int_{\Sigma_{0}} \exp (i \lambda(x-y) \xi)(i \lambda)^{-k} \\
& \quad \times p_{k}(x, \xi, \lambda) v(y, \lambda) d y \wedge d \xi, \\
& \Sigma_{0}: \text { contour d'intégration, } \quad \xi=-\operatorname{Im} x+i C(\bar{x}-\bar{y}), \\
& |x-y|<r, \quad C \gg 1, \quad 0<r C \ll 1, \quad M \gg 1 .
\end{aligned}
$$

Selon Lebeau [14, Proposition 4.2.1 dans la page 1469 et Théorème 4.2.1 dans la page 1472] et sa preuve, on obtient la proposition suivante:

Proposition 4.2.1. (Lebeau [14]) Soit $p, q \in \mathscr{E}_{V, m, \gamma}^{+}, \gamma>0, w \in H_{\varphi_{0}}\left(\bar{\Omega}_{0} \times U\right)$, qui est à croissance, avec $\left|a_{0}\right|, b_{0}, c_{0}$ suffisamment petits. On suppose qu'il existe $a_{1} \in\left(a_{0}, b_{0}\right)$ telle que l'on ait

$$
V_{0} \cap\left\{x \in \mathbf{C}^{3}: a_{0}<\operatorname{Re} x_{1}<b_{0}\right\} \cap S S_{\varphi_{0}}(w) \subset\left\{x \in \mathbf{C}^{3}: \operatorname{Re} x_{1} \geqq a_{1}\right\} .
$$

Alors, on a (i)-(v) suivants.

(i) [Unilatéralité] On a $V_{0} \cap\left\{x \in \mathbf{C}^{3}: a_{0}<\operatorname{Re} x_{1}<b_{0}\right\} \cap\left(S_{\varphi_{0}}\left(O p_{2}^{\gamma}(p) w\right)\right)$ $\subset\left\{x \in \mathbf{C}^{3}: \operatorname{Re} x_{1} \geqq a_{1}\right\}$.

(ii) [Propriété locale de la régularité Gevrey 3] Si $V_{0} \cap\left\{x \in \mathbf{C}^{3}: a_{0}<\right.$ $\left.\operatorname{Re} x_{1}<b_{0}\right\} \cap S S_{\varphi_{0}}(w) \subset\left\{x \in \mathbf{C}^{3}: a_{1} \leqq \operatorname{Re} x_{1} \leqq b_{1}\right\}$ avec $b_{1} \in\left[a_{1}, b_{0}\right)$, alors, pour tout $z \in \mathbf{C}^{3}$ vérifiant $\operatorname{Im} z_{1}=0, b_{1}<\operatorname{Re} z_{1}<b_{0}$, il existe un voisinage $\omega_{2} \subset \mathbf{C}^{3}$ de $z, \delta>0, C>0$ tels que l'on ait

$$
\left|\left(O p_{2}^{\gamma}(p) w\right)(x, \lambda)\right| \leqq C \exp \left(\frac{\lambda}{2}\left|\operatorname{Im} x^{\prime}\right|^{2}-\delta \lambda^{1 / 3}\right) .
$$

pour tout $x \in \omega_{2}$ et tout $\lambda \geqq 1$.

(iii) [Calcul symbolique] On a $V_{0} \cap\left\{x \in \mathbf{C}^{3}: a_{0}<\operatorname{Re} x_{1}<b_{0}\right\} \cap S S_{\varphi_{0}}(E w)$ $=\phi$, où $E=O p_{2}^{\gamma}(p * q)-O p_{2}^{\gamma}(p) O p_{2}^{\gamma}(q)$.

(iv) Soit $z_{1} \in \mathbf{C}^{3}$ près de $z_{0}$ vérifiant $z_{1} \notin V_{0}$. Alors, on a

$$
z_{1} \notin S S_{\varphi_{0}}\left(O p_{2}^{\gamma}(p) w-\widetilde{O p}_{1}(p) w\right) .
$$

(v) Soit $h$ symbole analytique formel à $m$, qui est une $3 \times 3$ matrice. Alors, on a

$$
V_{0} \cap\left\{x \in \mathbf{C}^{3}: a_{0}<\operatorname{Re} x_{1}<b_{0}\right\} \cap S S_{\varphi_{0}}\left(\widetilde{O p_{2}}(h) w-O p_{1}(h) w\right)=\phi .
$$


Remarque. Lebeau [14] a prouvé Proposition 4.2.1 en utilisant des arguments de la deuxième microlocalisation sur l'espace conormal de $V_{0}$. Voir [14, A.63 dans la page 1489-1493].

\section{\$5. Preuve de Théorème 3.2 et Théorème 1}

Nous suivrons ici l'argument de Lebeau [14, §1-§3]. Rappelons que dans $\S 3$ on a étudié l'opérateur $P_{1}$;

$$
\begin{aligned}
& P_{1}=\lambda^{-2}\left(\partial / \partial x_{4}\right)^{2}+O p_{1}\left(\ell^{(1)}\left(x_{4}, x, \xi, \lambda\right) I_{0}\right) \quad \text { avec } \\
& \ell^{(1)}\left(x_{4}, x, \xi, \lambda\right)=\sum_{k=0}^{\infty} \ell_{k}^{(1)}\left(x_{4}, x, \xi\right) \lambda^{-k} \quad \text { formellement. }
\end{aligned}
$$

Selon Lebeau $[14, \S 1]$ et l'hypothèse (H.2),

$$
-\xi_{4}^{2}+\ell_{0}^{(1)}\left(x_{4}, x, \xi\right)=0
$$

est équivalent à

$$
\begin{aligned}
& x_{4}+q\left(x, \xi, \xi_{4}^{2}\right)=0 \quad \text { avec } \\
& q\left(x, \xi, \xi_{4}^{2}\right)=\xi_{1}-e(x, \xi) \xi_{4}^{2}+O\left(\xi_{4}^{4}\right), \\
& q \quad \text { est réel si }(x, \xi) \in \Lambda_{\varphi_{0}}(X), \quad \xi_{4} \in \mathbb{R} .
\end{aligned}
$$

Remarque. La notation $q$ en (5.2) est différente de celle en (2.1) dans $\S 2$.

Soit $\Psi\left(x, \xi_{4}, y, \eta\right)$ la solution des équations d'Hamilton-Jacobi:

$$
\left\{\begin{array}{l}
\frac{\partial \Psi}{\partial \xi_{4}}=q\left(x, \frac{\partial \Psi}{\partial x}, \xi_{4}^{2}\right) \\
\left.\Psi\right|_{\xi_{4}=0}=(x-y) \eta
\end{array}\right.
$$

Alors, on a

$$
\Psi\left(x, \xi_{4}, y, \eta\right)=\Psi_{0}\left(x, \eta, \xi_{4}\right)-y \eta \quad \text { avec } \quad \Psi_{0}\left(x, \eta, \xi_{4}\right)=\Psi\left(x, \xi_{4}, 0, \eta\right) .
$$

Comme Lebeau $[14, \S 2]$ a étudié, $\Psi$ entraîne la transformation canonique singulière

$$
\chi_{0}: \Lambda_{\varphi_{0}}(X) \rightarrow \Lambda_{\psi_{0}}(X)
$$

où $\psi_{0}$ est la fonction à valeur réelle de classe $C^{1,1}$, c.-à-d. fonction dérivable à dérivée lipschitzienne, dans $X$. En plus, $\psi_{0}$ est strictement pluri sousharmonique hors de $\left\{x \in X: \operatorname{Im} x_{1}=0\right\}$ et vérifie 


$$
\begin{gathered}
\chi_{0}\left[\Lambda_{\varphi_{0}}(X) \cap\left\{(x, \xi) \in T^{*} X: \xi_{1}=0\right\}\right]=\Lambda_{\psi_{0}}(X) \cap\left\{(x, \xi) \in T^{*} X: \xi_{1}=0\right\}, \\
\chi_{0}\left(j_{\varphi_{0}}\left(x_{0}\right)\right)=j_{\psi_{0}}\left(x_{0}\right) \quad \text { et } \quad \psi_{0}(y)=\varphi_{0}(y) \quad \text { si } \operatorname{Im} y_{1} \leqq 0 .
\end{gathered}
$$

Comme Lebeau [14, 2.21 dans la page 1449], on introduit les fonctions $A(x, \xi), B(x, \xi)$ en posant

$\left\{\right.$ Valeurs critiques $\left.\left[\xi_{4} \rightarrow \Psi_{0}\left(x, \xi, \xi_{4}\right)\right]\right\}=x \xi-B(x, \xi) \pm(2 / 3) A(x, \xi)^{3 / 2}$.

On définit $\rho(x, \xi)$ par

$$
A(x, \xi)=\xi_{1} \rho(x, \xi) .
$$

Pour $t \in \mathbf{C},\left(z ; t^{2}, \eta^{\prime}\right) \in T^{*} X$, on définit $F\left(z ; t, \eta^{\prime}\right)$ par

$$
F\left(z ; t, \eta^{\prime}\right)=B\left(z ; t^{2}, \eta^{\prime}\right)+\frac{2}{3} t^{3} \rho\left(z ; t^{2}, \eta^{\prime}\right)^{3 / 2} .
$$

Soit $h\left(x_{4}, x, y, \eta, \xi_{4}, \lambda\right)$ un symbole analytique classique, qui est $3 \times 3$ matrice, près de $x_{4}=0, x=y=x_{0}, \quad \eta=-\operatorname{Im} x_{0}, \quad \xi_{4}=0$. On note $J_{h}$ l'opérateur intégral

$$
\begin{aligned}
& \left(J_{h} f\right)\left(x_{4}, x, \lambda\right)=\int_{\xi_{4}^{-}}^{\xi_{4}^{+}} d \xi_{4} \int_{\Sigma_{0}} d y \wedge d \eta \\
& \exp \left(i \lambda\left(\Psi\left(x, \xi_{4}, y, \eta\right)+x_{4} \xi_{4}\right)\right) h\left(x_{4}, x, y, \eta, \xi_{4}, \lambda\right) f(y, \lambda)
\end{aligned}
$$

où

$$
\begin{aligned}
& \xi_{4}^{+}=i \delta_{0}+\delta_{1} \delta_{0}^{3 / 2}, \quad \xi_{4}^{-}=-\delta_{0}-i \delta_{0}^{2} \delta_{2}, \quad \delta_{0}, \delta_{1}, \delta_{2} \in \mathbf{R}, \\
& \Sigma_{0}: \text { contour d'intégration, } \quad \eta=-\operatorname{Im} x+i C(\bar{x}-\bar{y}), \\
& |x-y|<r, \quad C \gg 1, \quad 0<r C \ll 1, \quad|y| \leqq d_{1} \\
& f \in H_{\psi_{0}}\left(\left\{y:\left|y-x_{0}\right| \leqq d_{0}\right\}\right) \quad \text { avec } \\
& d_{1} \leqq D_{1} d_{0}, \quad C \geqq D_{1}^{-1}, \quad d_{1} C \leqq D_{0}, \\
& \left|\delta_{1}\right|<D_{0}, \quad 0<\delta_{2} \leqq D_{0}, \quad 0<\delta_{0}<D_{1} d_{1}, \\
& D_{0}, D_{1}: \text { constantes vérifiant } \quad D_{0} \gg D_{1}>0 .
\end{aligned}
$$

Selon Lebeau $[14, \S 3]$, on a

$$
J_{h} f \in H_{\varphi_{0}}^{+, \rho}\left(\left\{x:\left|x-x_{0}\right|<d_{2}\right\}\right)
$$

pour $d_{2}$ et $\rho$ suffisamment petits. En plus, on a

$$
S S_{\partial, \varphi_{0}}\left(J_{h} f\right) \subset \tilde{\pi} \circ F^{1}\left(\chi_{0}^{-1}\left(\Lambda_{\psi_{0}}\left(S S_{\psi_{0}} f\right)\right)\right)
$$

où $\tilde{\pi}$ est la projection définie au dessus de Définition 2.2 et il existe un symbole 
(scalaire) analytique classique $\sigma\left(x_{4}, x, y, \eta, \xi_{4}, \lambda\right)$ tel que

$$
\sigma\left(x_{4}, x, y, \eta, 0, \lambda\right) \equiv 1 \quad \text { et } \quad S S_{\partial, \varphi_{0}}\left(P_{1} J_{h} f\right)=\phi
$$

lorsque $h=\sigma I_{0}$.

On fixe $\tau>0$ suffisamment grand. On définit la fonction $\sigma_{1}^{\tau}\left(z, t, \xi^{\prime}, \lambda\right)$ par

$$
\sigma_{1}^{\tau}\left(z, t, \xi^{\prime}, \lambda\right)=(v+\tau)^{1 / 4} \exp \left(\frac{2}{3}\left((v+\tau)^{1 / 2}\left(v-\frac{\tau}{2}\right)-v^{3 / 2}\right)\right)
$$

avec $v=(\exp (i \pi / 3)) \lambda^{2 / 3} t^{2} \rho\left(z, t^{2}, \xi^{\prime}\right)$. On définit un opérateur d'intégral $I$ par

$$
\begin{aligned}
(I f)(y, \lambda)= & \int_{z_{1}^{-}}^{z_{1}^{+}} d z_{1} \int_{t^{-}}^{t^{+}} 2 t d t \int_{\Sigma^{\prime}} d z^{\prime} \wedge d \xi^{\prime} \\
& \exp \left(i \lambda\left(\left(y^{\prime}-z^{\prime}\right) \xi^{\prime}+\left(y_{1}-z_{1}\right) t^{2}+F\left(z, t, \xi^{\prime}\right)\right)\right) \\
& \times \sigma_{1}^{\tau}\left(z, t, \xi^{\prime}, \lambda\right)\left(T_{0} f\right)(z, \lambda)
\end{aligned}
$$

où

$$
\begin{array}{ll}
z_{1}^{+}=-z_{1}^{-} \in \mathbb{R}_{+}, \quad\left|z_{1}^{+}\right|=d_{3}, \quad \arg t^{-}=-\pi / 2, \quad \arg t^{+}=\alpha, \\
\left|t^{+}\right|=\left|t^{-}\right| \quad \text { avec } d_{3}, \quad \alpha>0 \quad \text { suffisamment petit, } \quad\left|t^{+}\right| \ll d_{3} \sin \alpha,
\end{array}
$$

le chemin d'intégration de $t^{-}$à $t^{+}$

reste dans $\{t \in \mathbb{C}: \arg t \in[-\pi / 2, \alpha]\}$,

$f \in H_{\varphi_{0}}\left(\left\{x:\left|x-x_{0}\right|<d_{4}\right\}, \quad 0<d_{3} \ll d_{4} \ll 1\right.$,

$\Sigma^{\prime}$ : contour d'intégration, $\quad \xi^{\prime}=-\operatorname{Im} y^{\prime}+i C\left(\overline{y^{\prime}}-\overline{z^{\prime}}\right)$,

$\left|y^{\prime}-z^{\prime}\right|<d_{3}, \quad C \gg 1, \quad 0<d_{3} C \ll 1$,

$T_{0}$ : tronquature définie à (4.2.2) dans $\S 4$ avec

$\left|a_{0}\right|,\left|b_{0}\right|,\left|c_{0}\right|>0 \quad$ suffisamment petits.

Selon Lebeau [14, Proposition 3.2], on a

$$
\text { If } \in H_{\psi_{0}}\left(\left\{x:\left|x-x_{0}\right|<d_{5}\right\}\right)
$$

avec $0<d_{5} \ll 1$.

Soit $h\left(x_{4}, x, y, \eta, \xi_{4}, \lambda\right)$ un symbole analytique classique, qui est une $3 \times 3$ matrice, près de $x_{4}=0, x=y=x_{0}, \eta=-\operatorname{Im} x_{0}, \xi_{4}=0$. On définit l'opérateur $\tilde{J}_{h}$ par

$$
\tilde{J}_{h}(*)=\left.J_{h}(*)\right|_{x_{4}=0} .
$$


Remarque. Selon (5.12), $\tilde{J}_{h}$ en (5.17) est une quantification de la transformation canonique $\chi_{0}^{-1}$ en (5.4).

Soit $m=j_{\varphi_{0}}\left(x_{0}\right) \in T^{*} X$ et $V=\left\{(x, \xi) \in T^{*} X: \xi_{1}=0\right\}$. Alors, on a $m \in$ $V$. Selon Lebeau [14, 33 et $\S 4]$, il existe $\gamma>0$ et $\ell^{h} \in \mathscr{E}_{V, m, \gamma}^{+}$tels que l'on ait

$$
\tilde{J}_{h} I=O p_{2}^{\gamma}\left(\ell^{h}\right) \text {. }
$$

En plus, $\ell^{h}$ est elliptique dans $\mathscr{E}_{V, m, \gamma}^{+}$si $h=\sigma I_{0}$ avec $\sigma$ vérifiant (5.13). Voir Lebeau [14, Lemma 3.8 dans $\S 3$ et $(4.2 .23)-(4.2 .25)$ dans la page 1472].

On définit $K^{[0]}$ par

$$
K^{[0]}=\tilde{J}_{h} I \quad \text { avec } \quad h=\sigma I_{0}
$$

et définit $k \in \mathscr{E}_{V, m, \gamma}^{+}$par

$$
k=\ell^{h} \quad \text { avec } \quad h=\sigma I_{0}, \quad \sigma \text { vérifiant }(5.13) .
$$

Notons que $k \in \mathscr{E}_{V, m, \gamma}^{+}$est elliptique.

Soit $g \in H_{\varphi_{0}}(\{x:|x|<d\})$, à croissance, $d>0$ suffisamment petit. Selon $(5.11)-(5.13)$ et $[14, \S 3], J_{h} I g$ est la solution sortante de

$$
\left\{\begin{array}{l}
P_{1} w=0 \\
\left.w\right|_{\lambda_{4}=0}=K^{[0]} g
\end{array}\right.
$$

si $h=\sigma I_{0}$.

Soit $B_{J}^{[0]}, R_{J}, Q_{J}, j=1,2$ les opérateurs définis dans Définition 2.3 et $h\left(x_{4}, x, y, \eta, \xi_{4}, \lambda\right)$ un symbole analytique classique, qui est une $3 \times 3$ matrice, près de $x_{4}=0, x=y=x_{0}, \eta=-\operatorname{Im} x_{0}, \xi_{4}=0$. Puisque chaque élément de $R_{1}$ est opérateur différentiel par rapport à $x_{4}$ et opérateur pseudo-différentiel par rapport à $x \in X$, il existe un symbole analytique classique, qui est $3 \times 3$ matrice et que l'on notera $\operatorname{gr}(h)$, tel que

$$
R_{1} J_{h}=J_{\operatorname{gr}(h)} .
$$

Soit $g \in H_{\varphi_{0}}(\{|x|<d\})$, qui est à croissance, avec $S S_{\varphi_{0}}^{X}(g)$ suffisamment petit. Selon (5.21) et Stefanov-Vodev [21, §2], $\left(R_{1} Q_{1}+R_{2} Q_{2}\right) K^{[0]} g$ est solution sortante de

$$
\left\{\begin{array}{l}
M w=0 \\
\left.w\right|_{\imath_{4}=0}=B^{[0]} K^{[0]} g
\end{array}\right.
$$

où $B^{[0]}=B_{1}^{[0]}+B_{2}^{[0]}$.

Dans la suite, on considère l'opérateur $R$ comme 0 , par abus de la notation, lorsque pour tout $w \in H_{\varphi_{0}}(X)$ vérifiant $V_{0} \cap S S_{\varphi_{0}}(w) \subset\left\{x \in X: \operatorname{Re} x_{1} \geqq 0\right\}$ on a $V_{0} \cap\left\{x \in X: a_{0}<\operatorname{Re} x_{1}<b_{0}\right\} \cap S S_{\varphi_{0}}(R w)=\phi$. 


\section{Lemme 5.1.}

(i) Il existe $\gamma>0$ et $a \in \mathscr{E}_{V, m, \gamma}^{+}$tels que l'on ait

$$
B^{[0]} K^{[0]}=O p_{2}^{\gamma}(a)
$$

(ii) $a \in \mathscr{E}_{V, m, \gamma}^{+}$est elliptique.

Démonstration de Lemme 5.1. Soit $b=b^{0,1}+b^{0,2}$ avec $b^{0, n}$ défini par la somme formelle (2.8) dans $\S 2$.

(i) Rappelons que

$$
B^{[0]} K^{[0]}=B_{1}^{[0]} K^{[0]}+B_{2}^{[0]} K^{[0]}
$$

Soit $h=\sigma I$ avec $\sigma$ vérifiant (5.13). Selon la définition de $B_{1}^{[0]}$ et $K^{[0]}$, on a

$$
B_{1}^{[0]} K^{[0]}=\left.\left(R_{1} J_{h} I\right)(*)\right|_{x_{4}=0}=\left.J_{\operatorname{gr}(h)} I(*)\right|_{x_{4}=0}=\widetilde{J_{\operatorname{gr}(h)}} I .
$$

Donc, il existe $\gamma>0$ et $a^{(1)} \in \mathscr{E}_{V, m, \gamma}^{+}$tels que

$$
B_{1}^{[0]} K^{[0]}=O p_{2}^{\gamma}\left(a^{(1)}\right) .
$$

Soit $k$ définit par (5.20). Selon Proposition 4.2.1, on a

$$
\begin{aligned}
B_{2}^{[0]} K^{[0]} & =O p_{1}\left(b^{0,2}\right) O p_{2}^{\gamma}(k)=O p_{2}^{\gamma}\left(\mu\left(b^{0,2}\right)\right) O p_{2}^{\gamma}(k) \\
& =O p_{2}^{\gamma}\left(\mu\left(b^{0,2}\right) * k\right)=O p_{2}^{\gamma}\left(a^{(2)}\right)
\end{aligned}
$$

où $a^{(2)}=\mu\left(b^{0,2}\right) * k$. On définit $a$ par $a=a^{(1)}+a^{(2)}$. Alors, on obtient (5.24).

(ii) Soit $\omega_{3} \subset X$ voisinage ouvert de $x_{0}$, suffisamment petit. On fixe le point $v \in \omega_{3} \cap H^{1}$. Alors, près de $v$ on a

$$
B^{[0]} K^{[0]}=O p_{1}(b) \widetilde{O p_{1}}(k)=O p_{1}(b) O p_{1}(v(k))=O p_{1}(b \circ v(k)),
$$

où $b \circ v(k)$ est la multiplication ordinaire des symboles analytiques classiques formels. Voir Lebeau [13, III.2] ou Sjöstrand [20]. Selon (5.24) et Proposition 4.2.1, on a

$$
B^{[0]} K^{[0]}=O p_{2}^{\gamma}(a)=O p_{1}(v(a))
$$

près de $v$. Selon (5.29) et (5.30), on a

$$
v(a)=b \circ v(k) \text {. }
$$

Selon Stefanov-Vodev $[21, \S 2], b_{0}$, qui est symbole principal de $b$, est uniformément elliptique dans $\omega_{3} \cap H^{1}$. Puisque $k \in \mathscr{E}_{V, m, \gamma}^{+}$est elliptique, $b_{0} k_{0}$ est aussi uniformément elliptique dans $\omega_{3} \cap H^{1}$, si $\omega_{3}$ est suffisamment petit. Ici, $k_{0}$ est symbole principal de $k$. Alors, (5.31) nous permet de savoir que $a \in \mathscr{E}_{V, m, \gamma}^{+}$est elliptique. 
Démonstration de Théorème 3.2. On conserve les notations dans Théorème 3.2. Selon (5.23), on a

$$
\left.\left(\partial f / \partial x_{4}\right)\right|_{x_{4}=0}=\left(B^{[1]} K^{[0]}\right)\left(B^{[0]} K^{[0]}\right)^{-1} g,
$$

où $B^{[1]}=B_{1}^{[1]}+B_{2}^{[1]}$. Par le même argument précédent, on peut savoir qu'il existe $c^{(1)} \in \mathscr{E}_{V, m, \gamma}^{+}, \gamma>0$ tel que l'on ait

$$
B^{[1]} K^{[0]}=O p_{2}^{\gamma}\left(c^{(1)}\right) .
$$

Selon Lemme 5.1, Proposition 4.1.3 et Proposition 4.2.1-(iii), il existe $c^{(2)} \in$ $\mathscr{E}_{V . m, \gamma}^{+}, \gamma>0$ tel que l'on ait

$$
\left(B^{[0]} K^{[0]}\right)^{-1}=O p_{2}^{\gamma}\left(c^{(2)}\right) .
$$

On définit $c$ par $c=c^{(1)} * c^{(2)}$. Alors, on a

$$
\left.\left(\partial f / \partial x_{4}\right)\right|_{\lambda_{4}=0}=O p_{2}^{\gamma}(c) g .
$$

La conclusion (3.2) est maintenant déduite par (5.35) et Proposition 4.2.1(ii).

Démonstration de Théorème 1. Le fait (i) a déjà été prouvé par Théorème 3.2 avec la théorie de Sjöstrand [20] et $[1, \S 3]$. Selon (3.2), $\left.(\partial u / \partial n)\right|_{N}$ dans Théorème 1 est partiellement Gevrey 3 holomorphe en $\left\{\exp s H_{q}\left(\rho_{0}\right): s_{0} \leqq s \leqq\right.$ $\left.s_{1}\right\}$. Donc, on obtient le fait (ii) en tenant compte de Lemme 6.3 qui sera énoncé dans $\S 6$.

\section{§6. Rappels sur Gevrey Holomorphie Partielle}

Dans cette section, on fait quelques rappels sur Gevrey holomorphie partielle, dont la notion a déjà été écrite dans Bardos-Lebeau-Rauch $[1, \S 5]$. Cf. [12].

Soit $X \subset \mathbb{C}^{n}, n \geqq 2$ un ensemble ouvert et $\varphi$ la fonction scalaire qui est strictement pluri sous-harmonique en $X$. Soit $B \subset X$ une sous-variété analytique réelle. On suppose que $B$ est involutive par rapport à $-2 i \bar{\partial} \partial \varphi$ et que la codimension réelle de $B$ dans $X$ est 1 . On note $\tilde{B} \subset T^{*} X$ la réunion de la complexifiée des feuilles de $\Lambda_{\varphi}(B)$. Selon Lebeau [13, §III.5], il existe une unique fonction pluri sous-harmonique $\varphi_{B}$ en $X$ vérifiant $\Lambda_{\varphi_{B}}(X)=\tilde{B}, \varphi_{B}<\varphi$ en $X \backslash B$ et $\varphi_{B}=\varphi$ en $B$. Soit $w \in H_{\varphi}(X)$ et $m \geqq 1$. On définit 3 espaces $G_{m}^{ \pm}(w)$, $G_{m}($ w) comme ci-dessous.

$$
\begin{aligned}
& y \in G_{m}^{-}(w) \stackrel{\text { déf }}{\Longleftrightarrow}{ }^{\exists} U \subset X \text { voisinage de } y \text { t.q. }{ }^{\forall} \varepsilon>0 \text {, } \\
& { }^{\exists} K>0 \text { t.q. }{ }^{\forall} x \in U,{ }^{\forall} \lambda \geqq 1, \\
& |w(x, \lambda)| \leqq K \exp \left(\lambda \varphi_{B}(x)+\varepsilon \lambda^{1 / m}\right) .
\end{aligned}
$$




$$
\begin{aligned}
& y \in G_{m}^{+}(w) \stackrel{\text { déf }}{\Longleftrightarrow}{ }^{\exists} U \subset X \text { voisinage de } y,{ }^{\exists} \varepsilon>0, \\
&{ }^{\exists} K>0 \text { t.q. }{ }^{\forall} x \in U, \quad{ }^{\forall} \lambda \geqq 1, \\
&|w(x, \lambda)| \leqq K \exp \left(\lambda \varphi_{B}(x)-\varepsilon \lambda^{1 / m}\right) . \\
& y \in G_{m}(w) \stackrel{\text { déf }}{\Longleftrightarrow} \quad{ }^{\exists} U \subset X \text { voisinage de } y,{ }^{\exists} \varepsilon>0, \\
&{ }^{\exists} K>0 \text { t.q. }{ }^{\forall} x \in U,{ }^{\forall} \lambda \geqq 1, \\
&|w(x, \lambda)| \leqq K \exp \left(\lambda \varphi(x)-\varepsilon \lambda^{1 / m}\right) .
\end{aligned}
$$

On dit que $w$ est partiellement Gevrey $m B$-holomorphe à $y$ si $y \in G_{m}^{-}(w)$. On n'écrit pas $B$ lorsqu'il n'y a pas de confusion. On dit que $w$ est Gevrey $m$ à $y$ si $y \in G_{m}(w)$.

Énonçons maintenant 3 lemmes fondamentaux. Soit $w \in H_{\varphi}(X)$.

Lemme 6.1. On a $G_{m}^{-}(w) \cap G_{m}(w)=G_{m}^{+}(w)$.

Soit $\Gamma$ une feuille de $B$ et $\Gamma_{0}$ sous-ensemble connexe de $\Gamma$.

Lemme 6.2. Si $\Gamma_{0} \subset G_{m}^{-}(w)$, alors on a $\Gamma_{0} \cap G_{m}^{+}(w)=\phi$ ou $\Gamma_{0} \subset G_{m}^{+}(w)$.

Lemme 6.3. Si $\Gamma_{0} \subset G_{m}^{-}(w)$, alors on a $\Gamma_{0} \cap G_{m}(w)=\phi$ ou $\Gamma_{0} \subset G_{m}(w)$.

On peut obtenir la conclusion de Lemme 6.3 à partir de Lemmes 6.1 et 6.2 . Donc, il suffit de prouver Lemmes 6.1 et 6.2. Puisque la définition de $G_{m}^{ \pm}(w)$ et de $G_{m}(w)$ est invariante sous la transformation canonique (Cf. Lebeau $\left[13, \S\right.$ III.6,7]), on peut supposer que $B=\left\{z \in X: \operatorname{Im} z_{1}=0\right\}, \varphi(z)=|\operatorname{Im} z|^{2} / 2$, $\varphi_{B}(z)=\left|\operatorname{Im} z^{\prime}\right|^{2} / 2$. Ici, $z=\left(z_{1}, z^{\prime}\right) \in \mathbb{C} \times \mathbb{C}^{n-1}$. Alors, la conclusion de Lemme 6.1 et de Lemme 6.2 résulte de l'argument dans Okada $[17, \S 1]$. Voir aussi [1, Lemme 5.3 dans la page 106].

Quant à la preuve de Théorème 3.1 et de Théorème 1 , la sous-variété $B$ correspend à la région Glancing $G^{1}$, qui a été définie dans $\S 2$.

\section{References}

[1] Bardos, C., Lebeau, G. and Rauch, J., Scattering frequence and Gevrey 3 singularities, Invent. Math., 90 (1987), 77-114.

[2] Bardos, C., Masrour, T. and Tatout, F., Observation and control of elastic waves, (J. Rauch-M. Taylor eds.) Singularities and Oscillation, IMA Vol. Math. Appl., 91 (1997), 1-16.

[3] Eskin, G., General initial boundary problems for second order hyperbolic equations, $D$. Reidel. Co. Dordrecht, London (1981), 19-54.

[4] Friedlander, F. G. and Melrose, R. B., The wave front set of the solution of a simple initialboundary value problem with glancing rays. II, Math. Proc. Cam. Phil. Soc., 81 (1977), 97120.

[5] Hörmander, L., The analysis of linear partial differential operators I-IV, Springer. 
[6] Iwashita, M. and Shibata, Y., On the analyticity of spectral functions for some exterior boundary value problems, Glasnik Mate., 23 (1988), 291-313.

[7] Kawashita, M., Sur les solutions asymptotiqus de l'équation élastique (en japonais), Thèse de Maître à l'Université d'Osaka (1988).

[8] Lafitte, O., The kernel of the Neumann operator for a strictly diffractive analytic problem, Comm. P.D.E., 20 (1995), 419-483.

[9] - Second term of the asymptotic expansion of the diffracted wave in the shadow, Asymptot. Anal., 13 (1996), 329-359.

[10] - Diffraction for a Neumann boundary condition, Comm. P.D.E., 22 (1997), 319359.

[11] Lascar, B. and Lascar, R., Propagation des singularités Gevrey pour la diffraction, Comm. P.D.E., 16 (1991), 547-584.

[12] Lebeau, G., Deuxième microlocalisation à croissance, Séminaire Goulaouic-MeyerSchwartz (1982-1983).

[13] —, Deuxième microlocalisation sur les sous-variétés isotropes, Ann. Inst. Fourier Grenoble, 35 (1985), 145-216.

[14] —, Régularité Gevrey 3 pour la diffraction, Comm. P.D.E., 9 (1984), 1437-1494.

$[15 \mathrm{~W} \longrightarrow$, Propagation de singularité Gevrey pour le problème de Dirichlet, Advanced in microlocal analysis, NATO A.S.I. published by Reidel (Garnir ed.) (1986), 203-223.

[16] Melrose, R. B., Microlocal parametrices for diffractive boundary value problems, Duke. Math. J., 42 (1975), 605-635.

[17] Y. Okada, Second microlocal singularities of tempered and Gevrey classes, J. Fac. Sci. Univ. Tokyo Sect. IA. Math., 39 (1992), 475-505.

[18] Sato, M., Kashiwara, K. and Kawai, T., Hyperfunctions and pseudo differential equations, Lect. Notes Math., 287 Springer (1973).

[19] Sjöstrand, J., Propagation of analytic singularities for second order Dirichlet problems, Comm. P.D.E., 5 (1980), 41-94.

[20] - Singularités analytiques microlocales, Astérisque, 95 (1982).

[21] Stefanov, P. and Vodev, G., Distribution of the resonances for the Neumann problem in linear elasticity outside a strictly convex body, Duke Math. J., 78 (1995), 677-714.

[22] Taylor, M. E., Grazing rays and reflection of singularities of solution to wave equation, Comm. Pure. Appl. Math., 29 (1976), 1-38.

[23] Yamamoto, K., Singularities of solutions to the boundary value problems for elastic and Maxwell's equations, Japan J. Math., 14 (1988), 119-163. 
